\title{
Analysis on Standardization and Individualization of Nation Wear
}

\author{
Jun Zhang \\ Department of Film and Television Arts, Shanghai Publishing and Printing College, Shanghai, China \\ Email: cllapse@126.com
}

How to cite this paper: Zhang, J. (2018) Analysis on Standardization and Individualization of Nation Wear. Art and Design Review, 6, 96-107.

https://doi.org/10.4236/adr.2018.62009

Received: May 20, 2018

Accepted: May 28, 2018

Published: May 31, 2018

Copyright $\odot 2018$ by author and Scientific Research Publishing Inc. This work is licensed under the Creative Commons Attribution International License (CC BY 4.0).

http://creativecommons.org/licenses/by/4.0/

\section{(c) (i) Open Access}

\begin{abstract}
As one of the physical carriers of the national spirit, nation wear bears the important responsibility of cultural heritage and development, which are also the outer demonstration of the recognition of national identity, emotion and awareness. Based on the appreciable trends of standardization of the images and the production of nation wear, with reference to the successful experience of promoting standardization of nation wear in the international arena, this study puts forward the tentative plan of coding nation wear according to parameters like the pattern, component and No. and building the product database and national standard with the informationalized methods. On the other hand, to satisfy the individualized commands on the aesthetic value of the customers, this study suggests using the design philosophy of modules and carrying out the swift designs of changing the shapes with the standardized database of nation wear to realize the mass customization $(\mathrm{Mc})$ of nation wear.
\end{abstract}

\section{Keywords}

Clothes, Standardization, Nation Wear, Individualization

\section{Research Background}

With the 5000 years of splendid culture and history, China needs to use her own rich colors, exquisite techniques and unique styles to build Chinese clothes containing the profound culture to convey the Chinese civilization, which is not only the demonstration of nation's independence and esteem but also promoting the concepts of values of national identity, recognition and confidence through the culture of clothes.

The massive entrance of the western culture led to gradual absence of the Chinese clothes in people's daily lives in China. However, the 2008 Olympic 
Games and the 2010 Expo gave Chinese people the super opportunity to show soft strength and national characteristics of China to the world, which also give the chance to comprehensively promote standard nation wear with the symbolic significance of the country and the nation. Therefore, it's necessary to talk about the standardization of nation wear, the individualized development and the methods towards implementation. Graph 1 and Graph 2 are some traditional Chinese clothes.

As the card of clothes of culture, courtesy, art and traditions in Chinese nation, nation wear is expected to carry the unique cultural feature, the superb level of aesthetics, extraordinary techniques and rich pragmatic values in life. Meanwhile, nation wear in modern society need to combine the traditional elements and absorb different cultures, modern lives and work styles including the recognition of images in Chinese culture from home and abroad and the identity of the national spirit consisting of multi-ethnic groups.

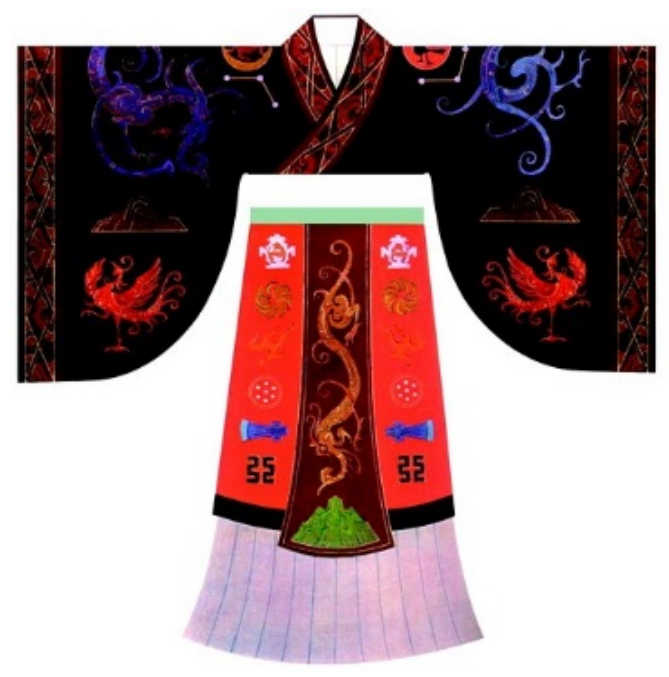

Graph 1. Traditional Hanfu.

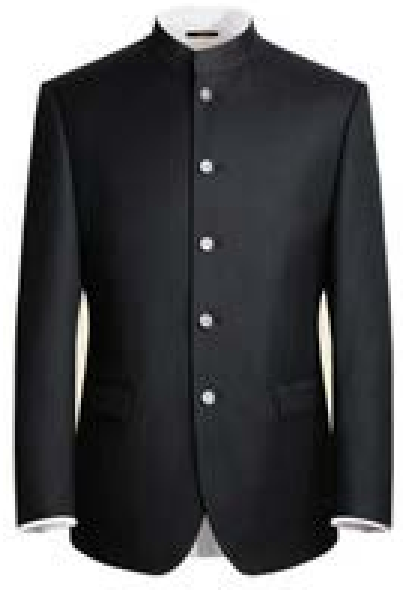

Graph 2. Chinese tunic suit. 
China is a multi-ethnic country with profound history and massive territory. Different eras, ethnic groups and regions have huge differences. So, what kind of clothes could best demonstrate the features of the whole Chinese nation? We need to carry out detailed and deep research on this issue.

The Asia-Pacific Economic Cooperation (APEC) led to the popularity of the clothes of Tang Dynasty. The dobby fabric, stand collar and Duijin fastener have become the relatively fixed styles till today. The clothes mainly focus on the dark red, wine red, sapphire and coffee with some colors like bright yellow and jade green. And the patterns on the fabric take the rotary flower mission with rich Chinese features as the main decorations or the Chinese characters of $\mathrm{Fu}, \mathrm{Lu}$ and Shou. As for the fabric, the clothes of Tang Dynasty usually choose the silk or brocade. Such mode of the clothes of Tang Dynasty not only inherits the styles and fabric full of traditional Chinese culture but also absorbs some modern draping skills.

As China dress, cheongsam is the widely recognized female clothes with the Chinese clothes styles in modern and contemporary times. Like the Indian Sari, kanga in East Africa and the draped skirt in Western Europe, cheongsam can strongly represent its regional culture. Han $\mathrm{Fu}$, which once became the default standard of the cultural system in the eastern clothes, exert a far-reaching impact on the mainstream clothes styles in neighboring countries within its long process of history. The emerging cultural innovation of appreciating the clothes of Han Fu among the educated people aims to carry forward the paofu (or shenyi) with crossing right lapel in addition to the clothes of Han Dynasty with long beizi. Such awareness of the national culture deserves the appreciation. However, the action of refusing the elements of Manchu and just recovering the clothes of Han Dynasty could be impartial. The history process of a nation must contain the combination with other cultures. In the early days, our ancestors have the broad mind taking the recognition of Chinese culture as the symbol rather than using the ancestry as the benchmark. Similarly, the development and evolvement of nation wear is the process of constantly absorbing the foreign cultures and making combination.

The clothes that represent the country are expected to be a match to the present productivity and the lifestyle. And the so-called tradition actually originated from the agriculture civilization. Even without the foreign factors, it's still necessary for adjusting the traditional clothes including the shortening and tightening of the long sleeves and robes, becoming the clothes featured with Chinese industrial civilization.

Under such circumstances of inheritance and development, to regulate the traditional clothes market and consciously standardize the images with prominent representatives, we need to classify nation wear into serials and make related national standards to facilitate the industrial manufacturing and promotion in large scale.

While absorbing the essence of the Chinese nation wear and keeping the traditional charm, nation wear should also use the new techniques and fabric to 
provide with Chinese clothes that are proper for the modern life so that people can choose individualized clothes. Such nation wear with both national characteristics and the standardized individualization could be favored by people from home and abroad.

\section{Research Content}

\subsection{Present Situation of Nation Wear}

Viewed from the present market, there's no national standard or industrial manufacturing for the traditional Chinese clothes. There are many well-educated people and foreign tourists buying and collecting nation wear from workshops. But nation wear can be applied to many areas including the engaged in the international exchange by officials, the important political and cultural situation and art ceremonies in additional to the other important folk occasions like traditional festivals, weddings, coming-of-age ceremony, commencement, birthday celebration, memorial ceremony, the individual social exchange and the cultural and art activities. Nation wear have gradually become the components of the daily clothes with huge market potentials.

Presently, the major subjects faced by nation wear in inheritance, development, innovation and promotion are as followings:

1) Uneven producing techniques and the relatively closed techniques of the craftsman.

The main producing method is masters teaching the students in the workshops. The disadvantages of single cutting, high cost, low efficiency and output are not appropriate in the assembly line and the commercial operation mode of the modern clothes, blocking the development and popularization of nation wear (Lu, 2003).

2) The information society emphasizes on high efficiency and automation.

The advanced digital technologies could facilitate the development of nation wear. But people lack the research and the innovation awareness including the standardization and the making garments in terms of nation wear for a long time.

3) The separation of manufacturing and research.

The separated operation and old techniques can't meet the increasing market demand for nation wear. Seizing the opportunity and liberalizing the operation methods to plan and sell nation wear could yield large economic results.

4) Abiding by the development rules of the culture, analyzing the essence and culture core of nation wear.

Carrying forward the innovation spirit and demonstrating individual requirements are the important work in inheriting and developing nation wear and making it the national image.

\subsection{References on the Standardization of Foreign Nation Wear}

\section{1) Kimono}


Kimono is the traditional Japanese clothes. You can always see elegant women wearing kimono walking on the Japanese streets; images or models in kimono in shop windows and counters. There are also different kinds of kimonos printed on the newspapers, magazines and publicizing materials. And even the small key chains, small bags and posters have the decorations like the kimono, clog, small umbrella and handbag. The popularity of kimono makes it the symbol of Japan. The traditional kimono has standard sizes as following: $158 \mathrm{~cm}$ of the clothes in length, $32 \mathrm{~cm}$ in width on the sleeve, $31 \mathrm{~cm}$ in width for the shoulder's part and $49 \mathrm{~cm}$ for the sleeve depth. The necessary articles or accessories for kimono have standard names such as jizhuo, changzhuo, changruban, zudai and fujin. The accessories have different serials and styles which are varied from occasions like wedding, banquet, mourning and ceremony and the four seasons. The flowers and the fabric of kimono can tell the social status of the people. According to different occasions, the kimono can be divided into yukata, furisode, tomesode, houmongi, bridal kimono, ordinary kimono and graduation kimono.

In addition to the franchise of the kimono, all kinds of malls and large supermarkets also have the counters as the selling channels for retailing or customizing. Some shops in the downtown of the city center also sell the fabric and all kinds of accessories of kimono for making kimono at home.

Recently, due to the limitation of wearing methods and occasions, less people are buying and using the traditional kimono. And the new kimono of yukata and haori are more popular. They shorten the length and simplify the accessories on the traditional basis. And the brocade is replaced by the cotton for decreasing the cost and facilitating the wearing. In addition, the washer wrinkle fabric is of unique attraction. These standardized new kimonos in garments with characteristics are similar in styles with the traditional kimono but with simple accessories and low price, which are popular among the young people. Graph 3 shows the samples of Japanese Kimono.

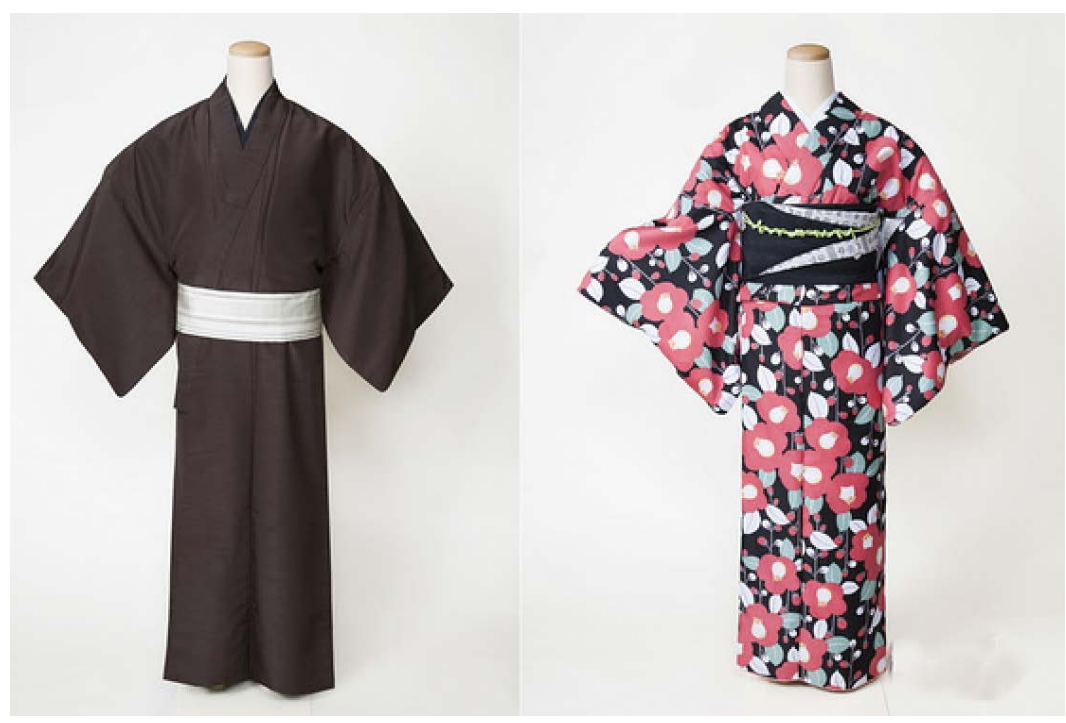

Graph 3. Samples of Kimono. 


\section{2) Hanbok}

Women's hanbok is jeogori with elegant chima. And men wear short magoja with long pants and the daenim tightening the bottom. Nowadays, even though many Korean are used to wearing the western clothes, some people are still in favor of the traditional Korean clothes on festivals like the Spring Festival and the Mid-Autumn day or the weddings.

The hanbok can be classified by identity, function, gender, age, application and material. In the modern perspective, the differentiation on the application can represent a lot. According to different occasions, the hanbok can be worn in wedding, the 60th birthday party and the 1st birthday party. Due to the complex process of wearing hanbok, it is only worn on the special day. Thus, the simple and convinent hanbok is designed for daily life. The hanbok for daily life have many kinds and different styles for the varied fabric and designs. It is also popular among the foreign tourists for its beauty and the low price.

The modern nation wear of Japan and South Korea have their own standards on criteria. Meanwhile, they inherit the traditional clothes rationally and combine the convenient requirements of the modern life. The multi choices on fabric and the solid cutting simplify the wearing process, focus on the market demand and the economic value and enlarge the range of the appropriate people. They combine the traditional culture and the development of the modern economy and society, realizing the customizing and comprehensively promotion of nation wear. Graph 4 shows the samples of Korean Hanbok.

\section{Application and Effect of the Research Results}

\subsection{The Preliminary Research on the Standardization of Nation Wear}

\section{1) The image standardization}

Referring to the experience of the standardization of nation wear of Japan and South Korea, China must regulate the style, color, pattern and quality of the fabric with the national standard to give clear and recognizable features of the complete image of nation wear to popularize the Chinese clothes.

The image standardization lies on the basis of the classification of nation wear. According to the target of the defining nation wear, it's proper to use the emerging time of the different nation wear in history as the criteria. The representative clothes are as followings: bianfu in Pre-Qin era, hufu in the period of Warring State, rufu in Han Dynasty and cheongsam in Qing Dynasty (Feng, 2012).

Discussing the style of nation wear, there are 4 features in the style of it: the first is the stand collar and the buttons in very middle of the front of the jacket; second, there is no seam between sleeves and jacket; third, the costumes are jackets with buttons down the front or in diagonal way; last, they have right-angled buttons. As for the fabric, the traditional national wear mainly uses brocade, silk, cotton, and linen. 


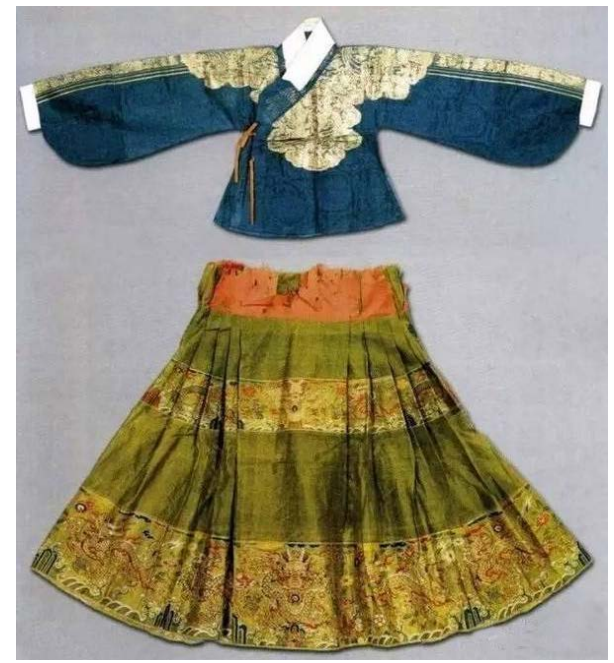

Graph 4. Samples of Hanbok.

The unified language in clothes is crucial for popularizing the culture of nation wear and regulating the market. Based on the classification standards of nation wear and the coding theory of components, we can make related standard language with national standard modes, which can be complementary strategies for the standardization of the image of nation wear.

\section{2) Manufacturing standardization}

The fact that China's manufacturing clothes occupying the top in the global trade for clothes proves that we have clothing capacity and huge potential market.

However, the requirements like the special wearing occasions, making and the fitness of nation wear lead to the situation imposed to nation wear with large ratio of customizing, small batch, short delivery period and severe competition in price. Thus, the manufacturing companies of nation wear must operate the standardized producing and regulate the management.

Building a set of detailed and regulatory files of basic techniques to provide the reasonable standards for manufacturing and reduce the uncertainties in the traditional management facilitates the informatization building of the company to form a reasonable and order production and management system of nation wear.

The design of the procedure flow diagram of nation wear is the generalization and the concrete reflection of the technique routes conveying the main procedures and equipment used in the producing process with the diagram. Due to varied kinds of products in nation wear with different raw and auxiliary materials and the different processing requirements, we need to get full understanding of the structure, technique requirements, producing methods and functions of the equipment to make the procedure flow so as to make it reasonable. Taking the cheongsam as an example, it has the features of varied styles, complex techniques and long processing time. Aimed at the features above, the standard technique flow diagram could facilitate realizing the management of the tech- 
nique flow and the integration of the information. In addition, building the standard procedures and measuring the standard labor hours with certain work load could facilitate the standardization of the production.

3) Information coding - the unavoidable way towards standardization of nation wear

It's imperative to develop the informatization work and regulate the products, accessories, cutting pieces, techniques and related terms and on which basis to code the national standard information code. Such method could facilitate the well-organized, high-efficient and fast operation and management for the products with digitalized technology to maximize the comprehensive competitive power.

The design philosophy and principle of the standard size of nation wear ( $\mathrm{Su}$, Qi, \& Gu, 2013).

The earliest theory system on building database through standard part design in CAD system is the German Standardization Institute (DIN) which is relatively complete.

The present general principles of the national standard part design GB1009.1-Definition and Principles of the Tabular layouts of article characteristics (GB/T15049 CAD Standard Part File) mainly refer to the DIN standards. Graph 5 is the case of China dress classification code.

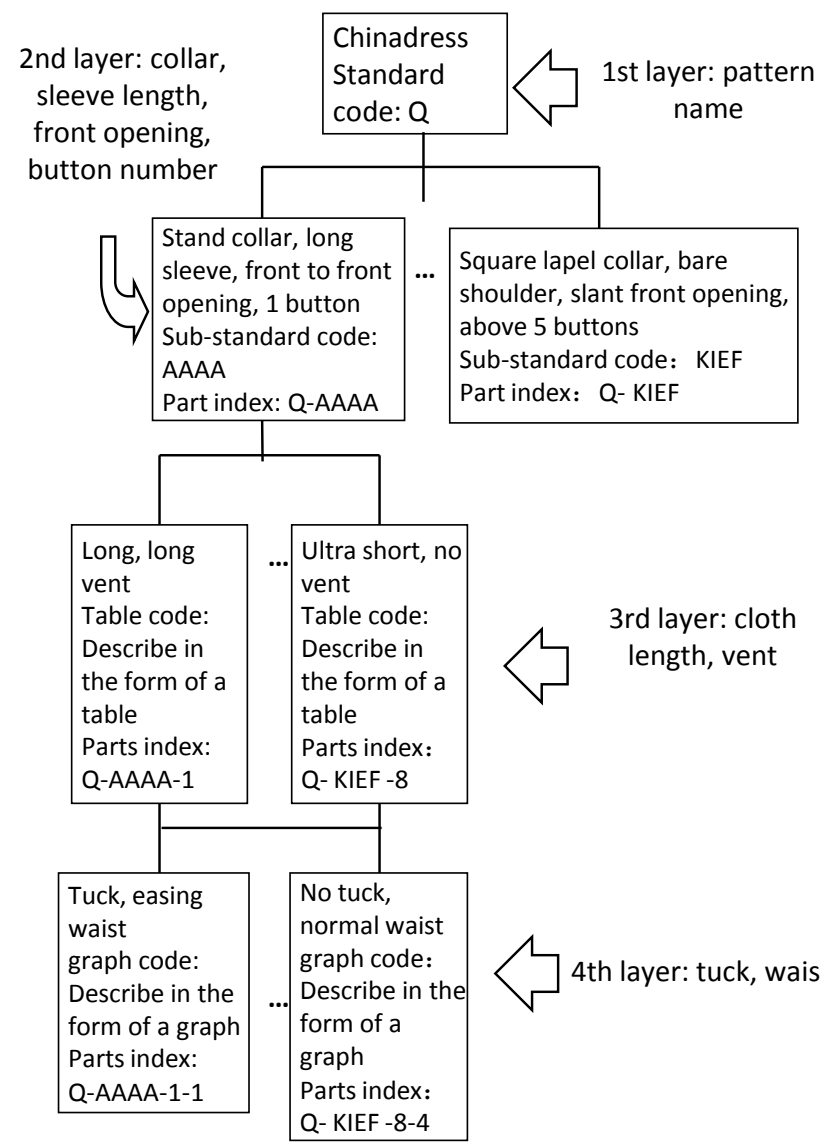

Graph 5. Case of style classification code (Chinese dress). 
This study suggests to make the category code proper for the massive customizing of nation wear and make supplement and revision if necessary according to the contents in DIN4000 (China National Standard GB/T 10091.1).

The design of coding information must follow the principles as: 1. giving full consideration to the need of seeking the same and similar products and parts in varied

\subsection{The Individualized Requirements of Nation Wear}

\section{1) The individualized appeal of the customers}

Nation wear needs to target at the common people rather than just the political officials, fashionable people, the middle-class and the white-collar workers who are pursuing high-quality life and admiring contents of culture and fashion.

Nowadays, the consumers are more rational in judging the fashion trend rather than blindly chasing and pursuing all kinds of trends. In comparison, consumers are showing the individualization in clothes consuming and attaching more importance to the clothes showing unique charm and style. Especially for the young generation, they are more acceptable for the new things with open character and bold feature to try.

The individualization of nation wear is the necessary methods to cater to the consuming trend, avoid the similar products and to maximize the yielding with non-price competition.

2) Calling of the times for exploration and innovation

Innovation can give new vitality and vigor to the Chinese clothes to realize the diversity of nation wear and the individualized development. And such innovation can be made in many aspects like the style, technique, fabric and color including breaking the traditional asymmetric patterns with the non-asymmetric cutting; combining and grafting the traditional patterns on the fabric to realize the exquisite match of the traditional and modern fabric and breaking the taboo of traditional color match with adjusting the contrast colors.

Only the national things could extend to the international arena. This study wishes that the Chinese clothes with innovation could better convey the beauty of Chinese clothes and spread the Chinese clothes culture. Graph 6 is the Nation wear style customize design process

\section{Application Prospect of the Results}

With the expanding of the market, consumers have increasing expectation for the styles, fabric and the delivery date. And the producing method of workshop is not only fit for nation wear. The development of nation wear should not only meet the needs of diversity and individualization of the customers but only keep the high-efficiency and low cost of the mass production. It's predictable that the mass customization could be the best solution towards this issue.

\subsection{Mass Customization (MC)}

With the advantages of large-scale producing and the fast speed, the mass 


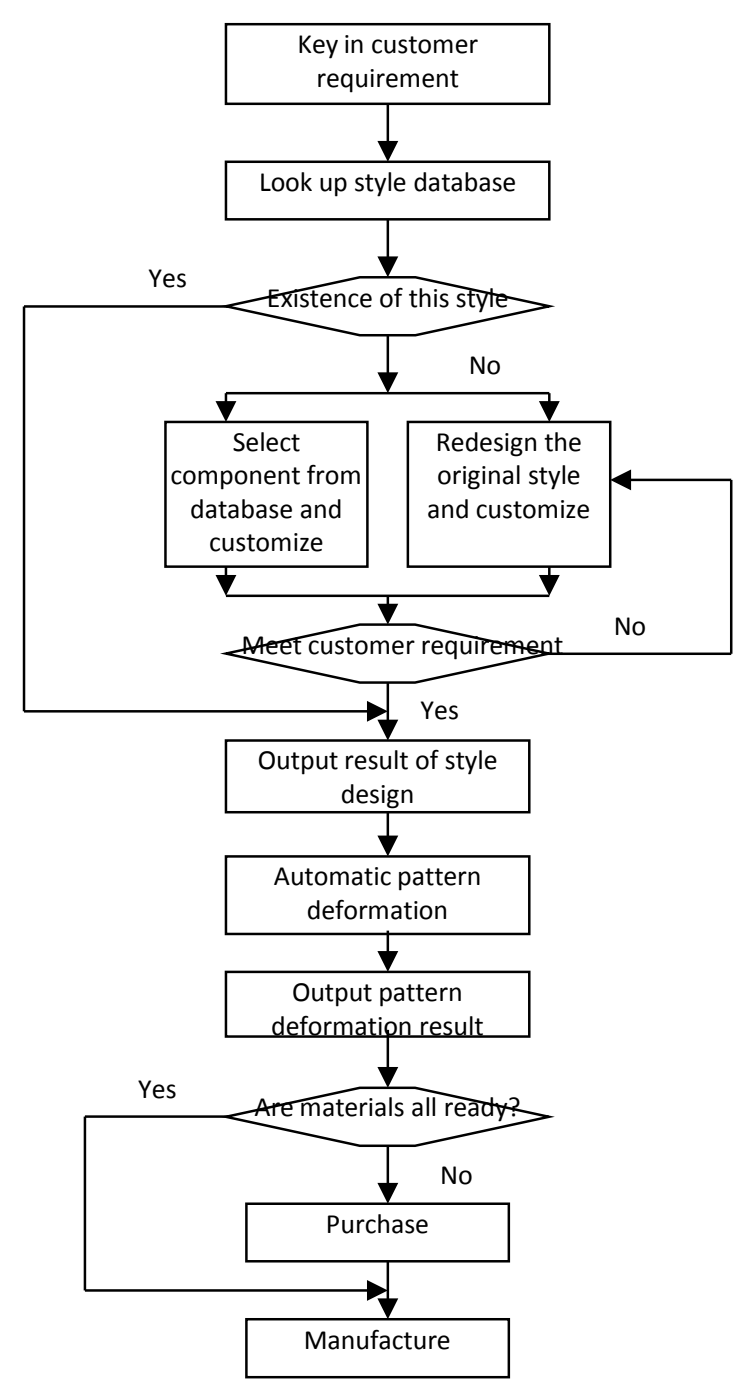

Graph 6. Nation wear style customize design process.

customization provides with individualized products at any amount for individual customer or market with special need through customization.

The core of MC is to divide the design of the products into two levels to satisfy the universal modular design for the certain needs of the period and the modified modular design for special needs of the customers. Taking the cheongsam as an example, the universal modular design classify the elements like the style, part and fabric for the specialized large-scale manufacturing; And on such basis, the exclusive modified module (such as embroidery modular, accessory modular ) is supplemented to satisfy the customer with special needs.

\subsection{Setting Parts Database}

MC could generate conflicts between the external diversity and internal diversity and the disputes between the customization production and the economic performance. One of the purposes of developing the modular is to increase the similarity and the reusability in the products and procedures. During the standar- 
dization, the modular design needs to response to varied kinds by sticking to a fundamental principle and to satisfy the individualized needs of customers with the least changes. The library of style, part, sample and fabric after the modularization are the bridges between the development of mass customization and design.

The design process of nation wear mainly includes customization design of the style, modified design of the sample and the choices of the fabric and accessory. The customization design process is the bilateral exchange process with the modular library. After receiving the orders, one needs to find the proper style in the modular library and customize it according to the requirements of the customer. After customizing the style, the modified design is the next step referring to the body shape of the customer. And the last step is the marking design. The style customization, sample modification and the fabric and accessory choice could be saved into the modular library for the repeated application.

Thus, there should be rational kinds of styles in customizing the clothes. And the internal diversity needs to reflect the individualized diversity of the customer. Both the setting design and the modified design need the support of the modularization.

\subsection{Setting Parts Database Technology of Fast Modification Design}

The modified design of clothes make changes on the fundamental style to satisfy the individualized need of the customer. The concept of modified design of clothes originated from the old days such as making sample yardage (also known as grading). With the application of the modularization design, the extent of the modified design is extended to the modified structure and style.

Nowadays, with the popularizing of the application of CAD and CAM in clothes, the process of designing nation wear from the beginning to the sample and the final garment could be made and the modified design is easier. For ordinary orders, we just need to use the data of the basic style in the modular library like the collars, length of the sleeve, the front part and fasteners and make the modified design according to the fabric and attached information. As for those orders with special requirements on shape, style, processing technique or the fabric, a specific person is expected to trace the process and understand the right implementation of the order in time after modifying the basic style. As an emerging software of managing date, the Product Data Management (PDM) can management the coding, technique, scale, size and the procedures of nation wear. When there is change in the main model, the related parts or the main model of the style should change accordingly and generate related technique order automatically. Such function is crucial to mass customization for only small amount of adjustments in the values of the qualities and characteristics of the parts or the shape could get access to the whole customized technique files automatically. 
Taking the cheongsam as an example, PDM could manage the data of marking and producing according to the modified sample aimed at different styles and fabric to make the final products.

It's predictable that in the coming future, the Chinese nation wear could carry on the liberalization operation like other clothes with reference to the national standard gaining the recognition of the majority. The mass customization and the fast modification after modularization could be a reasonable choice for producing nation wear.

\subsection{Expectation}

With the economic and social development of our country, the national strength increases with times. Chinese people are full of expectation and aspiration to seek the origin of the national culture and rejuvenate the Chinese nation. Such desire of seeking the origin of our culture would increase with the strengthening of the national status. In the trend of returning to and rejuvenating the traditional Chinese culture, the exploration of the rich national spirit and the unique national strait of the Chinese nation will definitely rejuvenate.

With the developing vision of keeping up with the times and the modern information methods, we can combine the inheritance and development, the standard and individualization organically to comprehensively promote the brilliant traditional culture of nation wear. And we have a promising future in realizing the industrial manufacturing and mass customization of the Chinese clothes so as to promote the seeding, growing and yielding of the tradition Chinese clothes with modern features.

\section{References}

Lu, J. C. (2003). Challenges and Saving Countermeasures of the Folk Culture of QianDongNan. Guizhou: Morden Guizhou.

Feng, Z. M., et al. (2012). Development History of Clothes. Beijing: China Textile Press.

Su, B. H., Qi, G. N., Gu, J. X., et al. (2013) Registration Principles of Accessory Library and the Application in Products Design. Beijing: Industrial Design Press. 